OPEN

SUBJECT AREAS:

ELECTRONIC PROPERTIES

AND MATERIALS

APPLIED PHYSICS

Received

31 October 2013

Accepted

11 December 2013

Published

10 January 2014

Correspondence and requests for materials should be addressed to

P.Z. (zhang_ping@ iapcm.ac.cn) or

G.-C.Z.

(zhang_guangcai@ iapcm.ac.cn)

\section{Morphology and growth speed of hcp domains during shock-induced phase transition in iron}

\author{
Wei-Wei Pang ' ${ }^{\text {, Ping Zhang }}{ }^{1,2}$, Guang-Cai Zhang ${ }^{1}$, Ai-Guo Xu' \& Xian-Geng Zhao'
}

\begin{abstract}
'Institute of Applied Physics and Computational Mathematics, Beijing 100088, People's Republic of China, ${ }^{2}$ Beijing Computational Science Research Center, Beijing 100084, People's Republic of China.
\end{abstract}

Emergence and time evolution of micro-structured new-phase domains play a crucial role in determining the macroscopic physical and mechanical behaviors of iron under shock compression. Here, we investigate, through molecular dynamics simulations and theoretical modelings, shock-induced phase transition process of iron from body-centered-cubic (bcc) to hexagonal-close-packed (hcp) structure. We present a central-moment method and a rolling-ball algorithm to calculate and analyze the morphology and growth speed of the hcp phase domains, and then propose a phase transition model to clarify our derived growth law of the phase domains. We also demonstrate that the new-phase evolution process undergoes three distinguished stages with different time scales of the hcp phase fraction in the system.

T he high-pressure states of iron have long been of great interest because of its technological and sociological importance as well as its geophysical role within Earth core ${ }^{1}$. In particular, the structural phase transitions induced by pressure and temperature have been extensively studied ${ }^{2}$. The structural phase transition of iron under shock loading was first reported by Bancroft in $1956^{3}$, where the transition pressure was determined as about $13 \mathrm{GPa}$ based on wave-profile analysis. After that, many research works have been done to investigate the transition pressure, transition mechanism and equation of state both in theory and at experiment ${ }^{4-6}$. In 2002, Kadau et al. first observed from large-scale molecular dynamics (MD) simulations the evolution process of phase transition from bcc into hcp structure in iron ${ }^{7}$. Later, they also studied the shocked polycrystalline iron ${ }^{8}$. In 2005, using the in situ X-ray diffraction technique with nanosecond resolution, Kalantar et al. directly confirmed the phase transition mechanism of iron'. That is, the bcc-hcp phase transition includes uniaxial collapse along the [001] direction and shuffling of alternate (110) planes of atoms. Using the same in situ technique combined with a modified Warren-Averbach method, in 2008, Hawreliak et al. derived the conclusion that single-crystal iron becomes nanocrystalline in shock transforming from bcc to hcp phase ${ }^{10}$, in reasonable agreement with results from large-scale MD simulations.

In addition to its microscopic mechanism, clearly, a physical modeling of phase transition process for shocked iron also crucially requires a deepened knowledge about the nucleation rate, growth speed, and the associated morphology evolution of nanoscaled new-phase domains (here, hcp domains), which keep unsolved up to now. This becomes particularly important when considering the above-mentioned experimental fact ${ }^{10}$ that shock transformed iron is really characterized by nanoscale grains. Inspired by this observation, through systematic MD simulations and a rationalized theoretical analysis, here, we study the non-equilibrium phase transition process in iron under the critical pressure. For this purpose we develop a central-moment method and a rollingball algorithm to calculate and analyze the morphology and growth speed of the single hcp phase domain. Then, we propose a phase transition model to help understanding our derived domain growth law. Finally, we demonstrate that the evolution process of hcp phase follows a three-stage description with different time scales of the hcp phase fraction in shocked iron.

\section{Results}

The sample material we use in simulations is single-crystal bcc iron. The simulation tool is the well-known LAMMPS software package ${ }^{11}$. The interatomic interaction is described by an embedded atom method (EAM) potential $^{12,13}$, which has been proved to be able to successfully describe the mechanical properties and structural phase transition behaviors of iron under high pressure. The simulation box consists of $240 \times 80 \times 80$ unit cells and contains approximately $3 \times 10^{6}$ atoms. The $x, y, z$ axes are along the [100], [010] and [001] directions, 
respectively. The periodic boundary conditions are applied along the $y$ and $z$ directions to minimize the surface and edge effects. The shock wave compression is generated using the momentum mirror method along the $x$ axis $^{14}$. In order to study the non-equilibrium phase transition process in detail, the shock velocities are chosen in between 300 and $400 \mathrm{~m} / \mathrm{s}$, which produce pressures around the critical value for phase transition. The velocity interval between two successive simulations is $10 \mathrm{~m} / \mathrm{s}$.

To identify the crystal structures, the atomic coordination numbers and common neighbor analysis (CNA) values are calculated. The single phase domain atoms are extracted using the cluster identification method. The phase interface shape is determined by our developed rolling-ball algorithm ${ }^{15}$. The growth speed of the phase domain is calculated using the central moment method and rollingball algorithm.

Figure 1 shows the evolution snapshots from bcc to hcp structure during the shock process. Figures 1(a)-(e) show the phase transition mechanism. Here, the green and blue spheres denote the upper and lower layers of atoms, and the red and yellow spheres mean that the

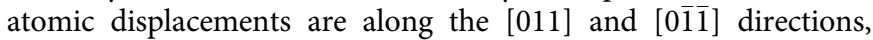
respectively. After the shock wave swept samples along the [100] crystalline orientation, the atoms are compressed along shock direction and form hexagons in the (011) and (01) planes, as shown in Fig. 1(b). Soon afterwards, some local atoms move through collective thermal fluctuations along [011] or [011] direction, as shown in Fig. 1(c). There is a relative slide between the two layers of atoms, which causes that the distance between each atom in the layers and its two second-neatest neighboring atoms along the $y$ and $z$ axes becomes farther. Once the defects form, they will drive the slip planes to slide alternately. The alternative slippage leads the bcc structure to evolve into the hcp structure and the phase transition process is finished, as shown in Figs. 1(d) and 1(e).

Figures 1(f) and 1(g) exhibit the formed hcp phase domains in the shocked region, where the hcp atoms are shown in blue color and the boundary atoms are shown in red color. From Fig. 1(f), it is clear that the nucleation sites of hcp phase domains are randomly located. The initial morphology of the single phase domain is ellipsoid-like, see the inset to Fig. 1(f). There are only two kinds of phase domains in the system. One kind is to stack into ellipsoid-like along (01 $\overline{1})$ crystal

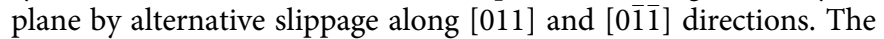
other kind is to stack into ellipsoid-like along (011) crystal plane by alternative slippage along the $[01 \overline{1}]$ and $[0 \overline{1} 1]$ directions. With the formation and growth of the hcp phase domains, different domains begin to interact and collide with each other. When the sliding types of two collided phase domains are the same, they link to form a bigger phase domain. If the sliding types of two collided phase domains are different, they form grain boundary and interact with each other, as shown in Fig. 1(g).

In general, once a phase domain core forms, it will gradually grow up through the outward movement of the phase interface. The growth speed depends on the driving force of phase transition and the synergic movement of interface atoms, which are closely related to the shape of phase interface. Therefore, the morphology evolution, growth speed, and the interactions among phase domains have always been a focus in the study of phase transition kinetics. In this work, we extract the single phase domain atoms, directly determine and visualize the phase interface shape by detecting surface atoms of the phase domain, as shown in Fig. 2, where panels (a)-(c) show the atomic evolution of the single phase domain, and panels (d)-(f) are the corresponding evolution of the phase interface shape. One can observe from Fig. 2 that the phase domain forms ellipsoid-like by a superposition of several layers of atoms. The growth of the phase domain mainly has two ways. The first way is the slide of each layer of phase plane, as shown in Figs. 2(a) and 2(b). The interfacial atoms surrounding the phase domain change into hcp arrangement via synergic movements. This type of growth is continuous. The second way is the successive activation process of new phase planes, as shown in Figs. 2(c), where two newly occurred phase planes are labeled as 13 and 14 in number. Compared to the slipping process of phase plane, the energy threshold for activating a new phase plane is higher, and an energy accumulation is needed. As a result, this type of growth is discontinuous.

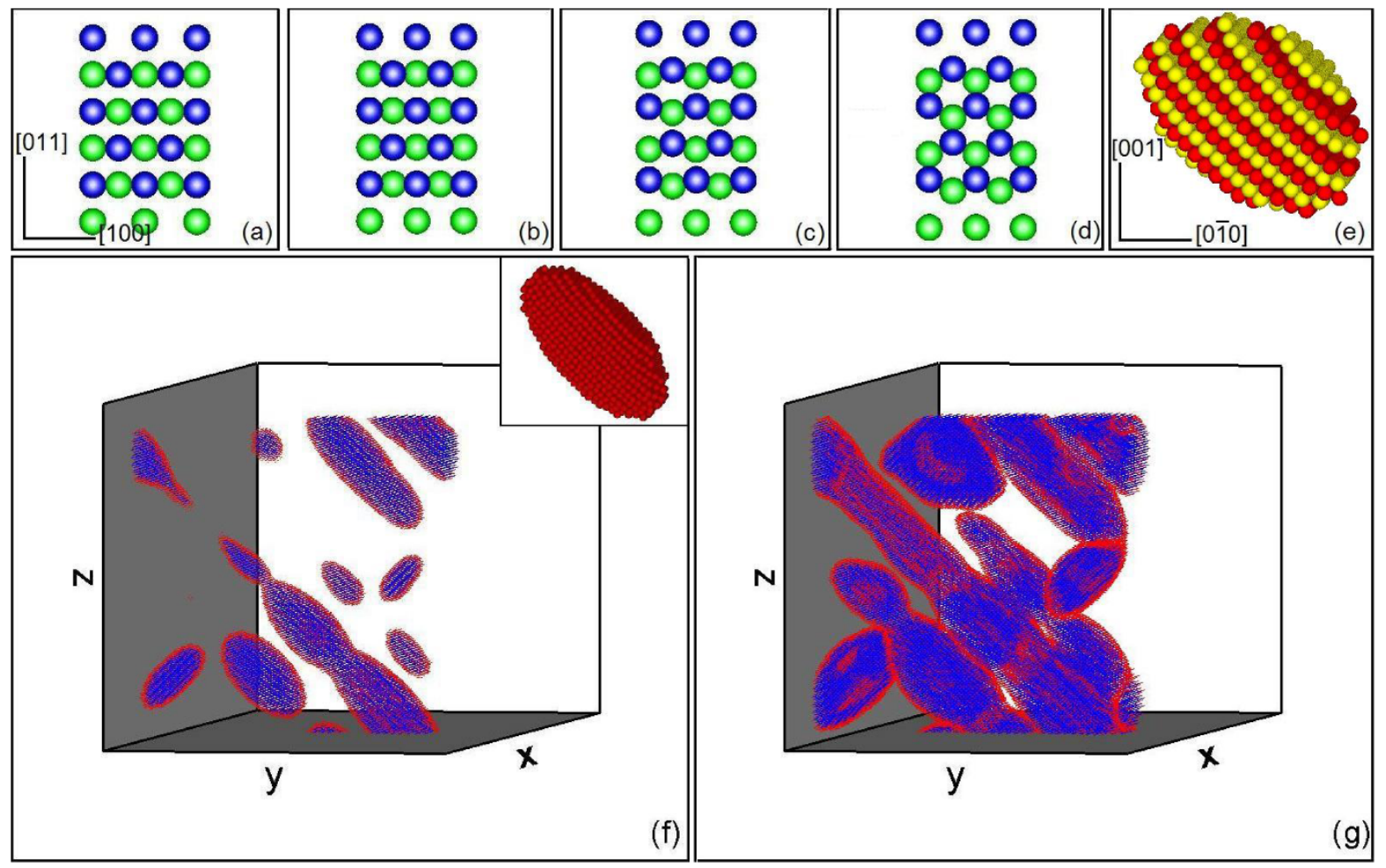

Figure $1 \mid$ Microstructure evolution in iron from bcc to hcp structure during the shock process. Panels (a)-(e) show the phase transition mechanism, while panels $(\mathrm{f})-(\mathrm{g})$ show the formed new phase domains in the shocked region. 


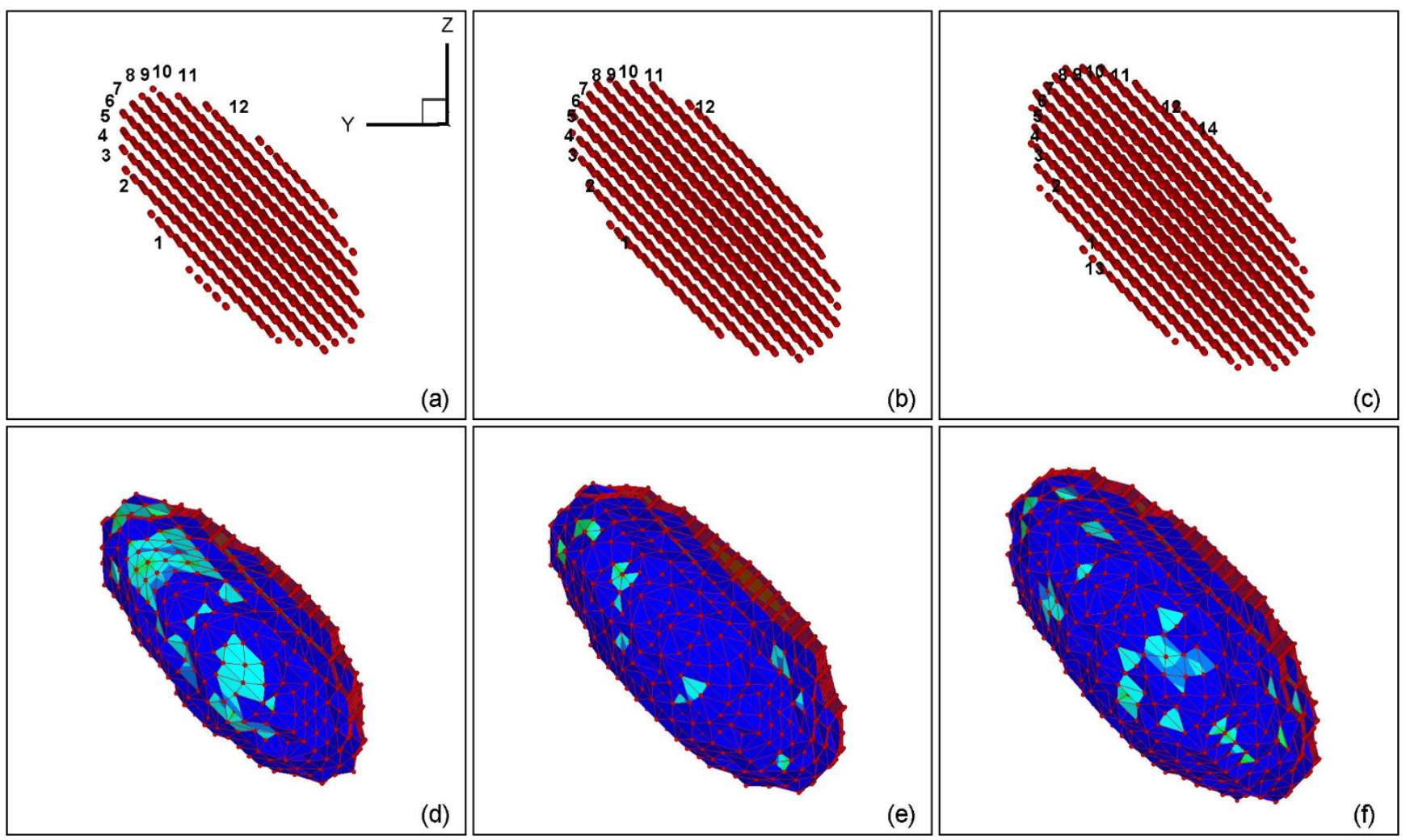

Figure $2 \mid$ Evolution snapshots of the single phase domain atoms and the corresponding phase interface shapes. Panels (a)-(c) are the atomic evolution of the single phase domain, while panels (d)-(f) are the corresponding phase interface shapes. Numbers in panels (a)-(c) denote layers of phase plane.

Based on its ellipsoidal-like appearance, the single phase domain can be reasonably approximated as an ellipsoid with uniform density. Their central moments should be the same. By comparing the eigenvalues and eigenvectors of central moments for the single phase domain and an ideal ellipsoid, we can determine the morphology parameters of the phase domain. The eigenvalues and eigenvectors of the central moment for the single phase domain satisfy

$$
\mathbf{A} \mathbf{x}_{i}=\mu_{i} \mathbf{x}_{i}(\text { for } i=1,2,3) .
$$

Here, the expression of the central moment $\mathbf{A}$ is given by

$$
\mathbf{A}=\sum_{i}^{n} m_{i}\left(\mathbf{r}_{i}-\overline{\mathbf{r}}\right)\left(\mathbf{r}_{i}-\overline{\mathbf{r}}\right),
$$

where $m_{i}$ and $\mathbf{r}_{i}$ are the mass and position of the $i$ th atom in the phase domain, respectively, and $\overline{\mathbf{r}}=\sum_{i}^{n} \mathbf{r}_{i} / n$ is the centroid. Whereas, the central moment of a uniform ellipsoid with the principal-axis lengths of $a, b$ and $c$ is given by

$$
\mathbf{A}=\iiint \rho \mathbf{r r} d \mathbf{V}=\frac{4 \pi \rho a b c}{15}\left(\begin{array}{ccc}
a^{2} & 0 & 0 \\
0 & b^{2} & 0 \\
0 & 0 & c^{2}
\end{array}\right),
$$

where $\rho$ is the atom density. Therefore, the three principal-axis lengths of the phase domain can be expressed as

$$
\left\{\begin{array}{l}
a=\sqrt[5]{\frac{15}{4 \pi \rho}} \sqrt[10]{\frac{\mu_{1}^{4}}{\mu_{2} \mu_{3}}} \\
b=\sqrt[5]{\frac{15}{4 \pi \rho}} \sqrt[10]{\frac{\mu_{2}^{4}}{\mu_{1} \mu_{3}}} \\
c=\sqrt[5]{\frac{15}{4 \pi \rho}} \sqrt[10]{\frac{\mu_{3}^{4}}{\mu_{2} \mu_{1}}}
\end{array}\right.
$$

In this paper, we calculate the central moment and principal-axis lengths according to the expressions (2) and (4) for multiple phase domains. The calculated results indicate that the three principal-axis directions (namely, three eigenvectors) approximately are [100], [011] and $[01 \overline{1}]$ for all phase domains. This demonstrates that the phase domain has different growth speeds along the shock loading direction, relative sliding and normal directions of phase planes. Figure 3 shows the principal-axis lengths and growth speeds of two phase domains which form at different times under the same shock velocity. One can observe that both the length and growth speed along the normal direction of phase plane are the smallest. The growth speed of the phase domain is prominently supersonic within a range $4 \times 10^{4}$ to $5 \times 10^{3} \mathrm{~m} / \mathrm{s}$. The time dependence of the principalaxis lengths can be approximately scaled as $L \sim t^{0.465}$ on average. In addition, we also note, from the growth speed curves along the [01 $\overline{1}]$ direction, remarkable oscillations with a period of $\sim 0.02 \mathrm{ps}$, which implies a discontinuous growth mode. The phenomenon is possibly caused by the fact that only the atomic layers exceeding a critical size are able to promote the activation of the new atomic layer. This is similar to the dislocation growth process, in which dislocation core should exceeds a critical size for the emission of dislocations.

Furthermore, based on the above results, we calculated and plotted the time evolutions of surface areas and volumes of the two phase domains according to the surface area and volume formulae of ellipsoid, as the red symbols shown in Fig. 4. To verify the reliability of our proposed central-moment method, we counted the numbers of total atoms and surface atoms for each phase domain (blue symbols in Fig. 4), and calculated the surface areas and volumes of phase domains (green symbols) using our developed rolling-ball algorithm. From Fig. 4, it can be observed that the calculated results by the central-moment and rolling-ball methods are consistent. This fact also confirms that the shape of the single phase domain is always close to be ellipsoidal at early time.

Theoretically, the evolution laws of surface area and volume of a single phase domain approximately follow $A \sim t^{m}$ and $V \sim t^{n}$, 

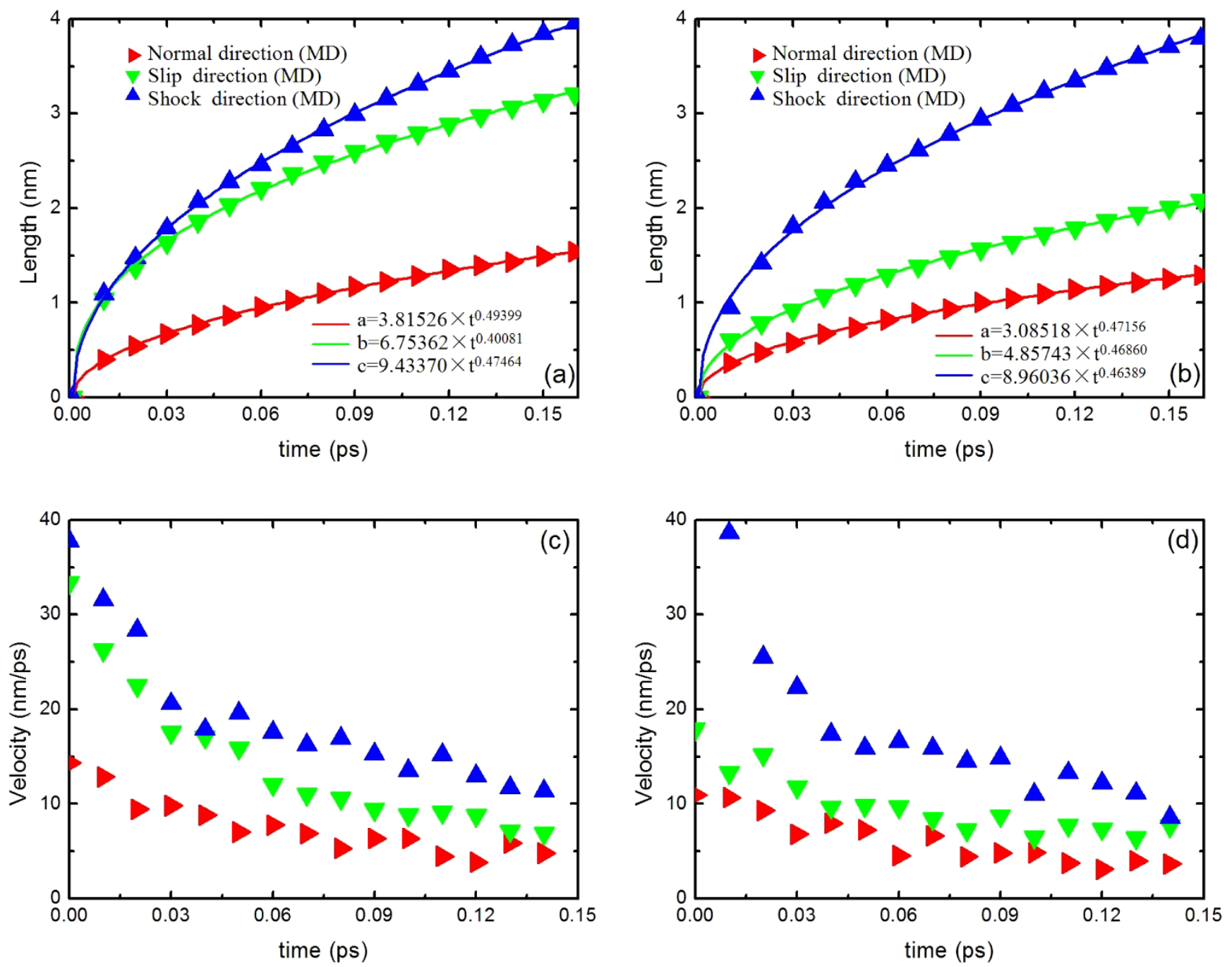

Figure 3 Principal-axis lengths and growth speeds of two hcp phase domains which form at different times under the same shock velocity. Panels (a) and (b) are the principal-axis lengths, while panels (c) and (d) are the growth speeds. The symbols are the MD simulated results and the lines are the fitting results.

respectively. If the principal-axis growth speed of the phase domain is constant, then $m=2$ and $n=3$. Otherwise, if the growth speed decreases with time, then $m<2$ and $n<3$. The black curves in Fig. 4 show our fitting results. We find that on average, the time evolution of the surface area and volume of a single phase domain can be respectively approximated as $A \sim t^{0.930}$ and $V \sim t^{1.395}$. The growth coefficients are almost invariant in the growth process of the single phase domain. But they are dependent of the nucleation times and positions of phase domains where the surrounding pressure are different.

As a complementary clarification, now we propose a phase transition model to illustrate the shocked kinetic process in iron based on the order parameter theory of Ginzburg-Landau. For this purpose we choose the slippage $(\xi)$ of the lattice as the order parameter, which varies from $\xi=0$ in bcc structure to $\xi=1$ in hcp structure, as schematically shown in Fig. 5(a), where the horizontal axis represents the distance away from the phase interface. In general, for the uniform (bulk) phase transition of iron, the system that undergoes transformation from bcc to hcp structure through the lattice slippage needs to overcome a potential barrier ${ }^{16}$, as schematically shown in Fig. 5(b). However, for the shocked iron, a solely uniform description is insufficient and the phase domain effects should be reasonably included in a phase transition model. In the nucleation stage of the phase domains, the atoms in the local region of stress concentration overcome the potential barrier by collective thermal fluctuations.
From above numerical results, we have obtained that in the growth stage of a phase domain, the growth speed is supersonic and the stress wave has no time to propagate in the hcp phase domain. Therefore, the growth of a phase domain is mainly driven by the interface energy. Figure 5(e) shows the potential energy distribution of a slice in the simulated system, where the regions of red, blue, and other colors represent the bcc, hcp, and interface structures, respectively. It is obvious that the potential energy in the interfacial region lies in amplitude between those in bcc and hcp regions. Thus, it is now clear that the interface energy is negative and prominently reduces the potential barrier between two phases, as schematically shown in Figs. 5(c) and 5(d). As a result, the transition process becomes easier.

With keeping this physical picture in mind, the energy of the system expressed with order parameters reads approximately

$$
F=\int\left[f(\xi)-\frac{D}{2}(\nabla \xi)^{2}\right] d^{3} \mathbf{r},
$$

where $f(\xi)$ and $-\frac{D}{2}(\nabla \xi)^{2}$ are the bulk free energy and interface energy of the system, respectively. $f(\xi)=\frac{a}{2} \xi^{2}-\frac{a+1}{3} \xi^{3}+\frac{1}{4} \xi^{4}$ is a bi-stable function with two stable points, $\xi=0$ and $\xi=1$. Here, the parameter $a$ is a system parameter related to temperature and pressure. Under low pressure, $a>1 / 2$, the bcc structure is stable, while under high pressure, $a<1 / 2$, the hcp structure is stable. The possible 

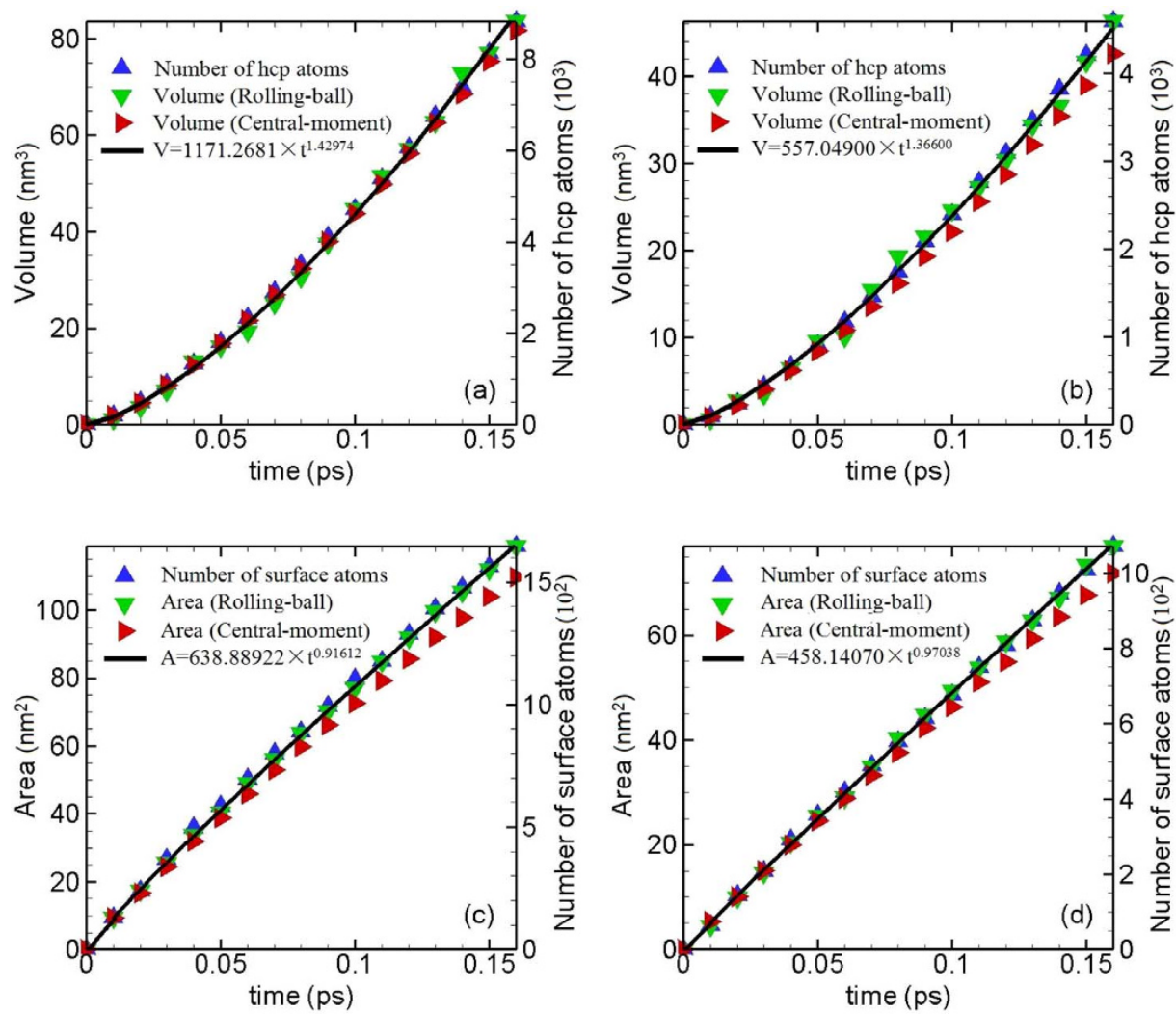

Figure $4 \mid$ Time evolutions of surface areas, volumes, and the corresponding atom numbers of the same two phase domains as those used in Fig. 3. The symbols are the MD simulated results and the lines are the fitting results.
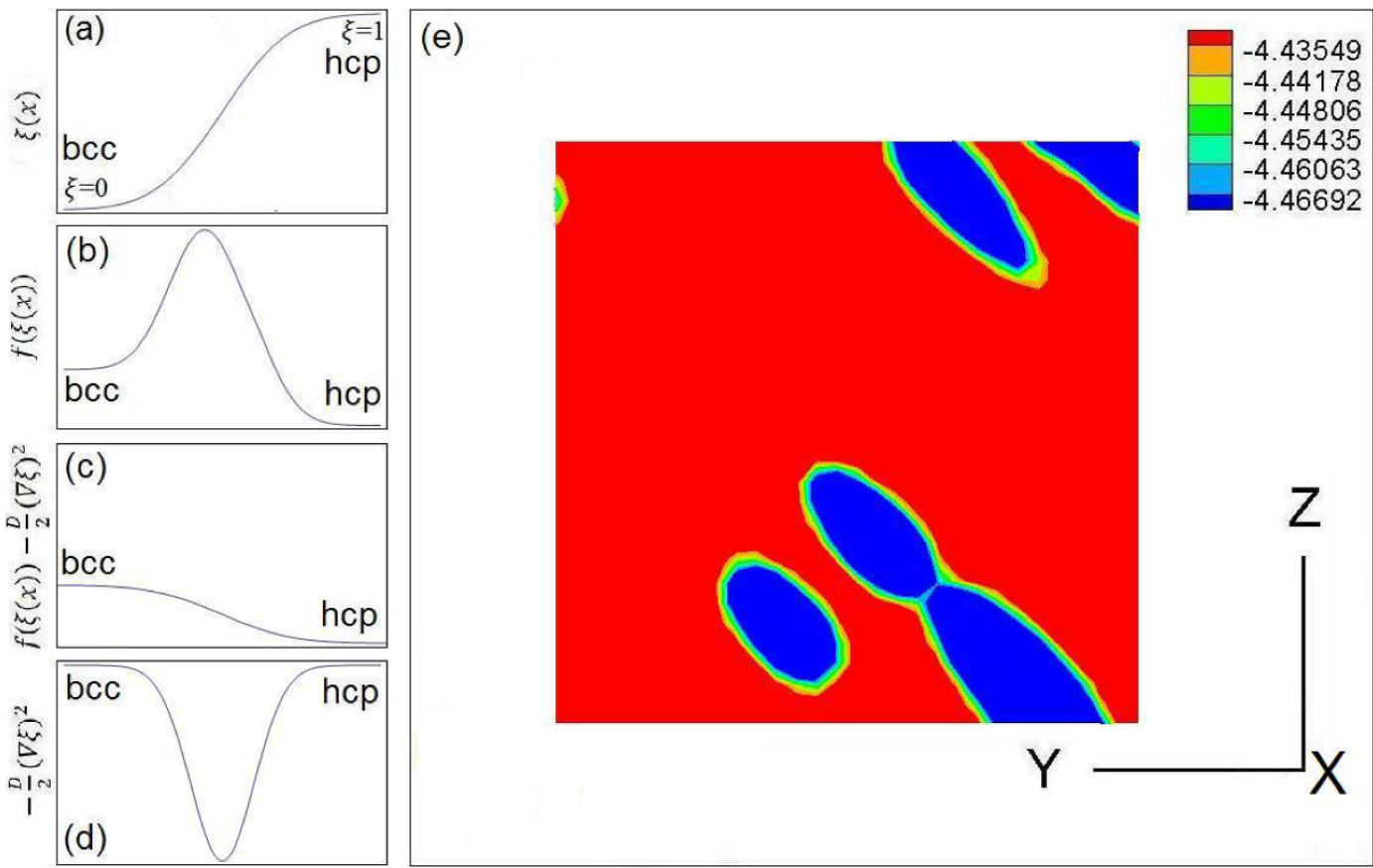

Figure $5 \mid$ The potential energy distribution and schematic diagram of phase transition. Panels (a)-(d) are the schematic diagram of phase transition, where the horizontal axis represents the distance away from the phase interface. Panel (e) shows the potential energy distribution of a slice in shocked iron. 
anisotropy in the interface energy has been ignored for simplicity. The evolution equation of the order parameter can be expressed as

$$
\partial_{t} \xi=\frac{\delta F}{\delta \xi}=f^{\prime}(\xi)-D \nabla^{2} \xi
$$

For the steady growth of one-dimensional phase domain, $\xi=\xi(\eta) \equiv$ $\xi\left(x-c_{0} t\right)$, and it satisfies the following eigenvalue equation

$$
\left\{\begin{array}{l}
f^{\prime}(\xi)-D \partial_{\eta}^{2} \xi+c_{0} \partial_{\eta} \xi=0, \\
\left.\xi\right|_{\eta \rightarrow-\infty}=0, \\
\left.\xi\right|_{\eta \rightarrow+\infty}=1,
\end{array}\right.
$$

where $c_{0}$ is the growth speed of the hcp phase domain. To describe the growth of a three-dimensional phase domain, we adopt the local coordinate system instead of the Cartesian coordinate, $\mathbf{r}=\mathbf{r}_{0}+\lambda \mathbf{n}$ $+\mu \mathbf{t}_{1}+v \mathbf{t}_{2}$, where $\mathbf{r}_{0}$ represents a point at the interface, and $\mathbf{n}, \mathbf{t}_{1}, \mathbf{t}_{2}$ represent the normal and two principal tangential unit vectors of the interface at the position $\mathbf{r}_{0}$, respectively. The evolution equation of order parameter can be rewritten as

$$
\partial_{t} \xi=f^{\prime}(\xi)-D\left(\partial_{\lambda}^{2}+\partial_{\mu}^{2}+\partial_{v}^{2}+\left(k_{1}+k_{2}\right) \partial_{\lambda}-k_{1} \partial_{\mu}-k_{2} \partial_{v}\right) \xi,
$$

where $k_{1}$ and $k_{2}$ are the curvatures along the two principal tangential directions, respectively. According to the relation $\xi=\xi(\eta) \equiv \xi(\lambda-$ $v t$ ), the above expression can be reduced to

$$
f^{\prime}(\xi)-D \partial_{\eta}^{2} \xi+(-D k+v) \partial_{\eta} \xi=0
$$

with the boundary conditions $\left.\xi\right|_{\eta \rightarrow-\infty}=0,\left.\xi\right|_{\eta \rightarrow+\infty}=1$, and $k=k_{1}$ $+k_{2}$. Comparing Eqs. (7) and (9), the growth speed of phase domain can be evaluated as

$$
v=c_{0}+D k \text {. }
$$

For shock-induced phase transition, the interface energy is related to the pressure surrounding the phase domain, and $D$ is a function of pressure. From above simulated results and theoretical analysis, we have obtained that the growth speed is supersonic and the $D$ is almost invariant in the phase domain growth process. Therefore, from Eq. (10) we get that the growth speed of the phase domain is a function of the local curvature. When the volume of phase domain is initially small, the local curvature is large and the energy that induces phase transition is relatively more concentrated. Thus, the growth speed is relatively high. With the growth of the phase domain, the interfacial area becomes much larger and the local curvature decreases, Therefore, the energy for phase transition becomes more dispersed and the growth speed decreases. This is consistent with our MD results.

For an ellipsoidal phase domain, the local curvature is nonuniform on the surface of the phase domain, and the larger the curvature, the higher the growth speed. Therefore, the phase domain becomes more and more flat or prolate with time. Actually, it has been shown in Fig. 1(g) that various phase domains have evolved to be disc-like, spherical, columnar, elongated, etc., in the later stage of shock loading. For the moment it is helpful to give a very simple but illustrative estimate on growth dynamics of the phase domain. For this purpose, if the phase domain is regarded as a sphere with radius $R$, the growth speed Eq. (10) reduces to $\dot{R}=c_{0}+2 D / R$. When hypothesizing $c_{0} \rightarrow 0$, one obtains that the linear length of phase domain is $R(t)=\sqrt{4 D t}$. Interestingly, our MD simulation results, which show that the linear length of an ellipsoidal phase domain is $L \sim t^{0.465}$, close to this analytical expression. The difference in the exponents $(0.465$ versus 0.5$)$ is caused by the difference in the curvatures between a sphere and an ellipsoid.

The material properties, such as the constitutive relation and equation of state, are significantly influenced by the transition fraction of the new phase ${ }^{17}$. In the non-equilibrium phase transition process under shock, the mechanical behaviors are coupled with the phase transition process. The phase transition fraction is relevant not only to the evolution of a single domain but also to the interactions among neighboring domains. Therefore, the phase transition fraction is a highly concerned quantity in the studies of material phase transition. Figure 6 (filled circles) shows the MD results of the time evolution of phase transition fraction under two different shock velocities. For every shock velocity, remarkably, there are three obvious stages, which accordingly represent different evolution characteristics of phase transition fraction. By comparing with atomic evolution images of phase transition process, we find that in the stage between points A and B (the first stage), new phase domains successively form and each phase domain independently grow up. In the stage between points $\mathrm{B}$ and $\mathrm{C}$ (the second stage), nearly no new phase domains form and the existing phase domains further grow up to interact with each other. In the stage after the point $C$ (the third stage), the phase domains get saturated in the perpendicular directions and grow up only along the shocking direction.

Theoretically, the phase transition fraction is a function of nucleation and growth rates of the phase domains. The nucleation number $I$ follows approximately $I \sim t^{m}$, while the atomic number $N$ of a single phase domain follows approximately $N \sim t^{n}$. In the case that both the nucleation of new phase domains and the growth of the existing phase domains simultaneously happen in the system, then the phase transition fraction $f$ follows approximately $f \sim t^{m+n}$. We have fitted our MD results, see Fig. 6. On average, we find that the evolution of phase transition fraction scales approximately $f \sim t^{1.89}$ in the first stage, $f \sim t^{1.23}$ in the second stage, and $f \sim t^{0.80}$ in the third stage.

\section{Discussion}

Shock-induced phase transition process of iron from bcc to hcp structure has been investigated via systematic molecular dynamic

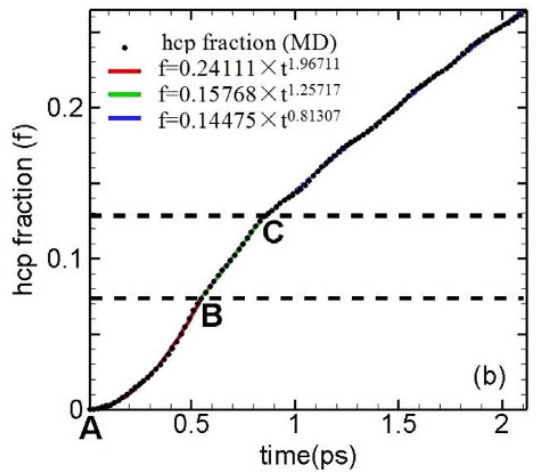

Figure 6 Phase transition fraction under two different shock velocities. Solid dots denote the MD results, while the red, green and blue lines represent the fitting results of three different evolution stages. The shock velocity is set at $380 \mathrm{~m} / \mathrm{s}$ in panel (a) and $400 \mathrm{~m} / \mathrm{s}$ in panel (b). 
simulations and theoretical analysis. Based on the shape characteristics of the hcp phase domains, we have proposed a central-moment method to calculate and analyze the morphology and growth speed of a single phase domain. It has been manifested that in the initial independent growth stage, the domain morphology is ellipsoid-like with three principal axes approximately along [100], [011], and [01 $\overline{1}]$ directions. The growth speed of a single phase domain is prominently supersonic within a range $4 \times 10^{4}$ to $5 \times 10^{3} \mathrm{~m} / \mathrm{s}$. We have shown that on average the size, surface area, and volume of the single hcp domain have their time evolutions $L \sim t^{0.465}, A \sim t^{0.930}, V \sim t^{1.395}$, respectively. Based on the order parameter theory of GinzburgLandau, we have presented a phase transition model to explain our found growth law of the single phase domain. Finally, we have demonstrated a three-stage evolution law for the phase transition fraction in shocked iron.

\section{Methods}

Three methods have been employed in our numerical simulations and data analysis: (i) The numerical MD simulations are performed using the well-known LAMMPS software package. The interatomic interactions used in the simulations are described by embedded atom method potentials. The shock wave compression is generated using the momentum mirror method; (ii) The atoms are distinguished by the common neighbor analysis (CNA) method. In this method the signature of the local crystal structure around a selected atom is identified by computing three characteristic numbers for each of the $N$ neighbor bonds of the central atom. The single phase domain atoms are extracted using the cluster identification method; (iii) The phase interface shape is visualized by the rolling-ball algorithm. The growth speed of the phase domain is calculated using the central-moment method and rolling-ball algorithm.

1. Stixrude, L., Cohen, R. E. \& Singh, D. J. Iron at high pressure: linearizedaugmented-plane-wave computations in the generalized-gradient approximation. Phys. Rev. B 50, 6442-6645 (1994).

2. Mao, H. K., Bassett, W. A. \& Takahashi, T. Effect of pressure on crystal structure and lattice parameters of iron up to $300 \mathrm{kbar}$. J. Appl. Phys. 38, 272-276 (1967).

3. Bancroft, D., Peterson, E. L. \& Minshall, S. Polymorphism of iron at high pressure. J. Appl. Phys. 27, 291-298 (1956).

4. Hasegawa, H. \& Pettifor, D. G. Microscopic theory of the temperature-pressure phase diagram of iron. Phys. Rev. Lett. 50, 130-133 (1983).

5. Boettger, J. C. \& Wallace, D. C. Metastability and dynamics of the shock-induced phase transition in iron. Phys. Rev. B 55, 2840-2849 (1997).

6. Wang, F. M. \& Ingalls, R. Iron bcc-hcp transition: local structure from x-rayabsorption fine structure. Phys. Rev. B 57, 5647-5654 (1998).

7. Kadau, K., Germann, T. C., Lomdahl, P. S. \& Holian, B. L. Microscopic view of structural phase transitions induced by shock waves. Science 296, 1681-1684 (2002).
8. Kadau, K. et al. Shockwaves in polycrystalline iron. Phys. Rev. Lett. 98, 135701 (2007).

9. Kalantar, D. H. et al. Direct observation of the $\alpha-\varepsilon$ transition in shockcompressed iron via nanosecond X-ray diffraction. Phys. Rev. Lett. 95, 075502 (2005).

10. Hawreliak, J. A. et al. High-pressure nanocrystalline structure of a shockcompressed single crystal of iron. Phys. Rev. B 78, 220101 (2008).

11. Plimpton, S. Fast parallel algorithms for short-range molecular dynamics. J. Comp. Phys. 117, 1-19 (1995).

12. Daw, M. S. \& Baskes, M. I. Embedded-atom method: derivation and application to impurities, surfaces, and other defects in metals. Phys. Rev. B. 29, 6443-6453 (1984).

13. Harrison, R., Voter, A. F. \& Chen, S. P. Atomistic Simulation Of Materials, edited by V. Vitek and D. J. Srolovitz (Plenum Press, New York, 1989), p.219-222.

14. Holian, B. L. \& Lomdahl, P. S. Plasticity induced by shock waves in nonequilibrium molecular-dynamics simulations. Science 280, 2085-2088 (1998).

15. Zhang, G.-C., Xu, A.-G. \& Lu, G. Molecular Interactions, edited by Aurelia Meghea (InTech Press, Croatia, 2012), p.361-400.

16. Liu, J. B. \& Johnson, D. D. Bcc-to-hcp transformation pathways for iron versus hydrostatic pressure: coupled shuffle and shear modes. Phys. Rev. B 79, 134113 (2009).

17. Mathon, O. et al. Dynamics of the magnetic and structural $\alpha-\varepsilon$ phase transition in iron. Phys. Rev. Lett. 93, 255503 (2004).

\section{Acknowledgments}

We acknowledge support of the National Natural Science Foundation of China under Grant No. 51071032, the National Magnetic Confinement Fusion Science Program of China under Grant 2012GB106001, and Science Foundation of CAEP under Grants No. 2011A0301016 and No. 2012B0101014.

\section{Author contributions}

W.W.P. did the calculations. P.Z., G.C.Z., A.G.X. and X.G.Z. analyzed the results. W.W.P., G.C.Z., and P.Z. wrote the paper. P.Z. and G.C.Z. were responsible for project planning and execution.

\section{Additional information}

Competing financial interests: The authors declare no competing financial interests.

How to cite this article: Pang, W.-W., Zhang, P., Zhang, G.-C., Xu, A.-G. \& Zhao, X.-G. Morphology and growth speed of hcp domains during shock-induced phase transition in iron. Sci. Rep. 4, 3628; DOI:10.1038/srep03628 (2014).

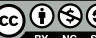

This work is licensed under a Creative Commons Attribution-

NonCommercial-ShareAlike 3.0 Unported license. To view a copy of this license, visit http://creativecommons.org/licenses/by-nc-sa/3.0 